Article

\title{
One Year after the Flood: Prevalence and Correlates of Post-Traumatic Stress Disorder among Residents in Fort McMurray
}

\author{
Wanying Mao ${ }^{1} \mathbb{D}$, Ejemai Eboreime ${ }^{1} \mathbb{D}^{\mathbb{D}}$, Reham Shalaby ${ }^{1} \mathbb{D}$, Nnamdi Nkire ${ }^{1}$, Belinda Agyapong ${ }^{1,2}$, \\ Hannah Pazderka ${ }^{1}$ (D) Gloria Obuobi-Donkor ${ }^{1}{ }^{D}$, Medard Adu $^{1}$, Ernest Owusu ${ }^{1}$ (D), Folajinmi Oluwasina ${ }^{1}$, \\ Yanbo Zhang ${ }^{1}$ and Vincent I. O. Agyapong ${ }^{1,3, *}$
}

check for

updates

Citation: Mao, W.; Eboreime, E.;

Shalaby, R.; Nkire, N.; Agyapong, B.; Pazderka, H.; Obuobi-Donkor, G.; Adu, M.; Owusu, E.; Oluwasina, F.; et al. One Year after the Flood: Prevalence and Correlates of PostTraumatic Stress Disorder among Residents in Fort McMurray. Behav. Sci. 2022, 12, 69. https://doi.org/ $10.3390 /$ bs 12030069

Academic Editor: Dario Siniscalco

Received: 20 January 2022

Accepted: 25 February 2022

Published: 2 March 2022

Publisher's Note: MDPI stays neutral with regard to jurisdictional claims in published maps and institutional affiliations.

Copyright: (C) 2022 by the authors. Licensee MDPI, Basel, Switzerland. This article is an open access article distributed under the terms and conditions of the Creative Commons Attribution (CC BY) license (https:// creativecommons.org/licenses/by/ $4.0 /)$.
1 Department of Psychiatry, University of Alberta, Edmonton, AB T6G 2B7, Canada; wmao2@ualberta.ca (W.M.); eboreime@ualberta.ca (E.E.); rshalaby@ualberta.ca (R.S.); nnamdi.nkire@albertahealthservices.ca (N.N.); bagyapon@ualberta.ca (B.A.); hannah@ualberta.ca (H.P.); obuobido@ualberta.ca (G.O.-D.); medard@ualberta.ca (M.A.); eowusu2@ualberta.ca (E.O.); folajinm@ualberta.ca (F.O.); yanbo9@ualberta.ca (Y.Z.)

2 Global Psychological E-Health Foundation, Edmonton, AB T6G 2B7, Canada

3 Department of Psychiatry, Faculty of Medicine, Dalhousie University, 5909 Veterans Memorial Lane, 8th Floor Abbie J. Lane Memorial Building, QEII Health Sciences Centre, Halifax, NS B3H 2E2, Canada

* Correspondence: vn602367@dal.ca; Tel.: +1-780-215-7771; Fax: +1-902-473-4887

\begin{abstract}
Background: The 2020 Fort McMurray (FMM) and area flood caused more than \$228 million in insured damage, affected over 1200 structures, and more than 13,000 people were evacuated. Objective: This study sought to determine the prevalence of post-traumatic stress disorder (PTSD)-like symptoms and the risk predictors among the population of FMM one year after the 2020 flooding. Methods: An online quantitative cross-sectional survey was distributed to residents of FMM via REDCap between 24 April to 2 June 2021 to collect sociodemographic, clinical, and flood-related information. The PTSD checklist for DSM-5 (PCL-C) was used to assess likely PTSD among respondents. Results: 186 of 249 respondents completed all essential self-assessment questionnaires in the analysis, yielding a response rate of $74.7 \%$. The prevalence of likely PTSD was $39.6 \%$ (65). Respondents with a history of depression were more likely to develop PTSD symptoms $(\mathrm{OR}=5.71 ; 95 \% \mathrm{CI}: 1.68-19.36)$. Similarly, responders with limited and no family support after the disaster were more prone to report PTSD symptoms ( $(\mathrm{OR}=2.87 ; 95 \% \mathrm{CI}: 1.02-8.05)$ and $(\mathrm{OR}=2.87$; 95\% CI: 1.06-7.74), respectively). Conclusions: Our research indicated that history of depression and the need for mental health counseling significantly increased the risk of developing PTSD symptoms following flooding; family support is protective. Further studies are needed to explore the relations between the need to receive counseling and presenting with likely PTSD symptoms.
\end{abstract}

Keywords: PTSD; trauma; flood; natural disaster; mental health; support; Fort McMurray

\section{Introduction}

Natural disasters have impacted many people worldwide throughout history and have always caused peaks in mortality and morbidity [1]. Since 1990, natural disasters have affected around 217 million people each year [2]; approximately 300 million people live with violent insecurity worldwide [1]. More than $43 \%$ of Canadians experienced a major disaster in their lifetime, 3\% reported being exposed to wildfire, and $1 \%$ had experienced major residential fires [2]. Over recent decades, natural disasters have occurred more frequently, and the scale has expanded more than ever before [1]. The reasons might boil down to increased urbanization rates, deforestation, environmental degradation, intensifying climate variables, such as higher temperatures, extreme precipitation, and more violent wind and water storms [1,3]. 


\subsection{Flooding and Mental Health Issues}

Floods account for approximately forty percent of natural disasters [4]. They are the most widespread weather-related natural disasters in developed and developing countries. Usually, they occur when an overflow of water submerges typically dry land [5]. Floods can happen following heavy rains, when huge ocean waves come on the shore, when the snow melts quickly, or when dams or levees collapse, and can last within minutes or over a long period (NSSL). The devastating impact of floods is evident in the floods in China (1959), Bangladesh (1974) and the tsunami in Southeast Asia in December 2004 [6]. In 2011, the Health Protection Agency (HPA) reviewed 48 research papers and those relevant government and non-government guidance documents published between 2004 and 2010, intended to assess and appraise the epidemiological evidence on flooding and mental health. The study indicated that flooding affects people of all ages, can exacerbate, or provoke mental health problems such as PTSD, depression, anxiety, panic disorder, etc., and highlights the importance of secondary stressors in prolonging the psychosocial impacts of flooding [7].

In the last two decades, studies have shown that the immediate impact of flood-related property damages, casualty and physical injuries may result in lasting mental health problems, including posttraumatic stress disorder (PTSD), depression, anxiety, and substance use disorders [3,8-13]. PTSD is among the most common post-disaster psychopathologies $[3,14]$. Research has documented the association between flooding and increased incidents of mental health illness, especially PTSD among survivors. A meta-analysis showed that among 40,600 flood victims, 3862 were diagnosed with PTSD after flooding; the combined incidence of PTSD was $15.74 \%$ [15]. PTSD symptoms and anxiety may last many years after the flooding $[16,17]$. A variety of risk factors increase the prevalence of PTSD symptoms in the aftermath of flooding, including socioeconomic factors [18], individual characteristics [19], mental health problem history [20], preparedness [21] and the severity of disaster $[18,22]$.

\subsection{Disaster Events and People in Fort McMurray}

Fort McMurray (FMM) is in the Regional Municipality of Wood Buffalo (RMWB) in Northern Alberta, Canada, with a diverse population of 111,687 in 2018 [23], mainly in temporary project accommodations and in adjoining rural communities [24]. On 26 April 2020, the RMWB declared a state of local emergency (SOLE) after flooding the northern Alberta city's downtown and surrounding areas, caused by a 25-kilometre-long ice jam formed in the Athabasca River [25]. This flood caused over \$228 million insured damages, destroyed 1200 structures and forced 13,000 residents to leave their homes [25]. At the same time, FMM was heavily affected by the COVID-19 pandemic [26]. The COVID-19 pandemic may profoundly affect an individual's physical and mental health [27,28]. Physical distancing and sanitation measures of COVID-19 also posted further challenges on evacuation, post-disaster regulation and recovery [29]. These physical and economic costs of such traumatic experiences always accompany emotional difficulties and stress [30] and dampen survivors' mental health and general well-being $[27,30,31]$. One example is FMM's wildfires associated with an evacuation order in 2016. The one-month prevalence of anxiety symptoms [32], PTSD [24], and depressive symptoms [24] were estimated at 19.8\%, 12.8\%, $14.8 \%$, respectively, six months after the wildfire [32].

As aforementioned, FMM experienced three major disasters in five years by May of 2021, including the wildfires in 2016, the flooding in 2020, and the COVID-19 pandemic in 2020. We hypothesize that the three disasters increased mental health problems. The accumulative effect of the disasters may have led to a higher prevalence of PTSD-like symptoms among FMM residents than the prevalence prior to before the flood. We aimed to examine the prevalence of PTSD among FMM residents one year after the flood, and the sociological and clinical risk factors of likely PTSD. The further purpose was to improve understanding of the significance of the recovery experience in mental health outcomes. 
This could lead to improved provision of support to those potentially most vulnerable to future mental health problems because of natural disasters such as flooding [16].

\section{Materials and Methods}

\subsection{Study Setting and Design}

Fort McMurray (FMM) is in the Regional Municipality of Wood Buffalo in Northern Alberta, Canada, with a diverse population of 111,687 as of the 2018 census [23]. Through a cross-sectional survey introduced from 24 April to 2 June 2021, an online questionnaire was distributed randomly via email to residents of FMM, Canada, via REDCap [33]. Government, school, occupation and community were used as platforms to collect data. Sociodemographic, clinical, flooding-related information (e.g., Did you lose property because of the floods in Fort McMurray? Did you live in the same house you lived in before the floods? etc.), and the level of support received from different jurisdictions (e.g., support received from family; support received from the insurer, etc.) were collected. All residents of Fort McMurray who received the online survey link could participate in this study. There were no exclusion criteria based on clinical or demographic criteria. Participants were provided with information about the survey, and informed consent was implied by completing the survey questionnaires. Study approval was granted by the University of Alberta Research and Ethics Committee (Pro00066054).

\subsection{Sample Size Estimation}

With a population of 111,687 as of the 2018 census [23], a $95 \%$ confidence interval, and a $\pm 3 \%$ margin of error, the sample size needed for prevalence rate estimates for PTSD will be 1058 .

\subsection{Outcome Measure}

The PTSD checklist civilian version (PCL-C) [34] was used to assess the presence or absence of likely PTSD in respondents randomly selected from the natural settings in FMM. Many studies indicated that the PCL-C demonstrated good internal consistency and retest reliability. Compared with alternative measures of PTSD, the PCL-C showed favorable patterns of convergent and discriminant validity. Therefore, it is suggested as a psychometrically valid tool for screening and assessing the severity of PTSD in clinical practice and research [35-38]. There were, in total, 17 questions; the options varied from 'significantly agree' to 'do not agree at all'. Patients with a PCL-C score of 44 or more were deemed to have likely PTSD [26,39,40].

\subsection{Statistical Analysis}

Data were analyzed using SPSS Version 25 [41]. Descriptive statistics were provided for demographic, clinical, and other variables against age. Chi-squared analyses were run to examine all the variables concerning the likely PTSD categorical variable. Logistic regression analysis was employed to identify significant risk factors of probable PTSD. We reported the responses without imputation of the missing data. Variables with a statistically significant relationship $(p \leq 0.05)$ or approaching significance $(0.05<p \leq 0.1)$ to a likelihood of PTSD on univariate analysis were included in a logistic regression analysis. Correlational analysis was performed to determine any strong intercorrelations (Spearman's correlation coefficient of 0.7 to 1.0 or -0.7 to -1.0 ) among predictor variables before applying logistic regression. Odds ratios from the binary logistic regression analysis were examined to determine the association between each variable in the model and the likelihood of respondents presenting with likely PTSD, controlling for the other variables.

\section{Results}

Among all the 249 participants who clicked on the survey link, 186 were returned with all key self-assessment questionnaires completed, giving a response rate of $74.7 \%$. Descriptive demographic, clinical and flood-related feedback was collected from the whole 
sample of participants $(\mathrm{N}=186)$, with age as a key variable (Table 1$)$. Overall, the mean age of our respondents was 42 years; the majority of the sample were female $85.5 \%$ (159); $71 \%$ (132) of the respondents reported that they were in a relationship; $94.1 \%$ (175) were employed; $48.4 \%$ (90) reported having no mental health diagnosis, while $64.5 \%$ (120) reported not receiving psychotropic medications; $38.7 \%$ (72) reported receiving mental health counseling, and $52.7 \%$ (98) reported that they would like to receive mental health counseling; 65 respondents reported likely PTSD symptoms, yielding a prevalence of PTSD in our population $(39.6 \%$; $95 \%$ confidence interval: $32.1-47.6)$.

Table 1. Demographic information, clinical characteristics and support received by respondents.

\begin{tabular}{|c|c|c|c|c|c|}
\hline Variables & $\begin{array}{l}\leq 30 \mathrm{y} \\
\mathrm{n}(\%)\end{array}$ & $\begin{array}{l}31-40 y \\
\text { n (\%) }\end{array}$ & $\begin{array}{l}41-50 y \\
n(\%)\end{array}$ & $\begin{array}{l}>50 y \\
\text { n }(\%)\end{array}$ & $\begin{array}{l}\text { Total } \\
\text { n }(\%)\end{array}$ \\
\hline \multicolumn{6}{|l|}{ Gender } \\
\hline Male & $4(12.9)$ & $5(8.8 \%)$ & $11(20.0)$ & $7(16.3)$ & $27(14.5)$ \\
\hline Female & $27(87.1)$ & $52(91.2)$ & $44(80.0)$ & $36(83.7)$ & $159(85.5)$ \\
\hline \multicolumn{6}{|l|}{ Employment status } \\
\hline Employed & $25(80.6)$ & $57(100.0)$ & $52(94.5)$ & $41(95.3)$ & $175(94.1)$ \\
\hline Unemployed & $6(19.4)$ & $0(0.0)$ & $3(5.5)$ & $2(4.7)$ & $11(5.9)$ \\
\hline \multicolumn{6}{|l|}{ Relationship status } \\
\hline In a relationship & $16(51.6)$ & $46(80.7)$ & $39(70.9)$ & $31(72.1)$ & $132(71.0)$ \\
\hline Not in a relationship & $15(48.4)$ & $11(19.3)$ & $16(29.1)$ & $12(27.9)$ & $54(29.0)$ \\
\hline \multicolumn{6}{|l|}{ Housing status } \\
\hline Own home & $20(64.5)$ & $41(71.9)$ & $42(76.4)$ & $38(88.4)$ & $141(75.8)$ \\
\hline Renting & $11(35.5)$ & $16(28.1)$ & $13(23.6)$ & $5(11.6)$ & $45(24.2)$ \\
\hline \multicolumn{6}{|l|}{$\begin{array}{l}\text { History of mental health diagnosis from } \\
\text { a health professional }\end{array}$} \\
\hline Depressive disorder & $9(29.0)$ & $18(31.6)$ & $20(36.4)$ & $11(25.6)$ & $58(31.2)$ \\
\hline Bipolar disorder & $0(0.0)$ & $2(3.5)$ & $3(5.5)$ & $1(2.3)$ & $6(3.2)$ \\
\hline Anxiety disorder & $11(35.5)$ & $28(49.1)$ & $27(49.1)$ & $12(27.9)$ & $78(41.9)$ \\
\hline Alcohol abuse & $1(3.2)$ & $0(0.0)$ & $1(1.8)$ & $1(2.3)$ & $3(1.6)$ \\
\hline Drug abuse & $0(0.0)$ & $0(0.0)$ & $1(1.8)$ & $1(2.3)$ & $2(1.1)$ \\
\hline Personal disorder & $0(0.0)$ & $1(1.8)$ & $1(1.8)$ & $0(0.0)$ & $2(1.1)$ \\
\hline Others & $3(9.7)$ & $7(12.3)$ & $4(7.3)$ & $3(7.0)$ & $17(9.1)$ \\
\hline No mental health diagnosis & $16(51.6)$ & $23(40.4)$ & $26(47.3)$ & $25(58.1)$ & $90(48.4)$ \\
\hline \multicolumn{6}{|l|}{ History of psychotropic medications } \\
\hline Antidepressants & $10(32.3)$ & $17(29.8)$ & $22(40.0)$ & $10(23.3)$ & $59(31.7)$ \\
\hline Antipsychotics & $1(3.2)$ & $1(1.8)$ & $2(3.6)$ & $0(0.0)$ & $4(2.2)$ \\
\hline Benzodiazepines & $2(6.5)$ & $1(1.8)$ & $0(0.0)$ & $1(2.3)$ & $4(2.2)$ \\
\hline Mood stabilizers & $2(6.5)$ & $3(5.3)$ & $4(7.3)$ & $3(7.0)$ & $12(6.5)$ \\
\hline Sleeping tablets & $5(16.1)$ & $4(7.0)$ & $7(12.7)$ & $5(11.6)$ & $21(11.3)$ \\
\hline Other & $0(0.0)$ & $1(1.8)$ & $1(1.8)$ & $1(2.3)$ & $3(1.6)$ \\
\hline Not on psychotropic medication & $20(64.5)$ & $37(64.9)$ & $33(60.0)$ & $30(69.8)$ & $120(64.5)$ \\
\hline $\begin{array}{l}\text { Respondents who received } \mathrm{MH} \\
\text { counseling in the past year }\end{array}$ & $18(58.1)$ & $24(42.1)$ & $19(34.5)$ & $11(25.6)$ & $72(38.7)$ \\
\hline $\begin{array}{l}\text { Respondents who would like to receive } \\
\text { MH counseling }\end{array}$ & $22(71.0)$ & 33 (57.9) & $29(52.7)$ & $14(32.6)$ & $98(52.7)$ \\
\hline $\begin{array}{l}\text { Respondents who resided at Fort } \\
\text { McMurray during the } 2020 \text { flood }\end{array}$ & $28(90.3)$ & $53(93.0)$ & $52(94.5)$ & $43(100.0)$ & $176(94.6)$ \\
\hline $\begin{array}{l}\text { Respondents who resided in the } \\
\text { flooding areas }\end{array}$ & 3 (10.7) & 8 (15.1) & $8(15.4)$ & $12(27.9)$ & 31 (17.6) \\
\hline $\begin{array}{l}\text { Respondents who witnessed the } \\
\text { flooding of homes or structures in For } \\
\text { McMurray }\end{array}$ & $18(69.2)$ & $42(77.8)$ & $37(72.5)$ & 34 (79.1) & $131(75.3)$ \\
\hline
\end{tabular}


Table 1. Cont.

\begin{tabular}{|c|c|c|c|c|c|}
\hline Variables & $\begin{array}{l}\leq 30 \mathrm{y} \\
\mathrm{n}(\%)\end{array}$ & $\begin{array}{l}31-40 y \\
n(\%)\end{array}$ & $\begin{array}{c}41-50 y \\
n(\%)\end{array}$ & $\begin{array}{l}>50 y \\
\text { n }(\%)\end{array}$ & $\begin{array}{l}\text { Total } \\
\text { n }(\%)\end{array}$ \\
\hline $\begin{array}{l}\text { Respondents who have been fearful for } \\
\text { their own lives, or the lives of their } \\
\text { close friends or family members }\end{array}$ & $6(23.1)$ & $19(35.2)$ & $16(31.4)$ & $10(23.3)$ & $51(29.3)$ \\
\hline \multicolumn{6}{|l|}{$\begin{array}{c}\text { During the } 2020 \text { Fort McMurray } \\
\text { flooding, how frequent did you watch } \\
\text { TV images about the devastation caused } \\
\text { by the flood? }\end{array}$} \\
\hline Daily & $11(42.3)$ & $35(64.8)$ & $40(78.4)$ & $30(69.8)$ & $116(66.7)$ \\
\hline$<$ Daily & $12(46.2)$ & $8(14.8)$ & $8(15.7)$ & $9(20.9)$ & $37(21.3)$ \\
\hline $\begin{array}{l}\text { I did not watch TV images about the } \\
\text { devastation }\end{array}$ & $3(11.5)$ & $11(20.4)$ & $3(5.9)$ & $4(9.3)$ & $21(12.1)$ \\
\hline \multicolumn{6}{|l|}{$\begin{array}{l}\text { During the } 2020 \text { Fort McMurray } \\
\text { flooding, how frequent did you read } \\
\text { newspaper and internet articles related } \\
\text { to devastation caused by the flood? }\end{array}$} \\
\hline Daily & $16(61.5)$ & $46(85.2)$ & $39(78.0)$ & $31(72.1)$ & $132(76.3)$ \\
\hline$<$ Daily & $9(34.6)$ & $7(13.0)$ & $10(20.0)$ & $9(20.9)$ & $35(20.2)$ \\
\hline $\begin{array}{l}\text { I did not read newspaper or articles about } \\
\text { the devastation }\end{array}$ & $1(3.8)$ & $1(1.9)$ & $1(2.0)$ & $3(7.0)$ & $6(3.5)$ \\
\hline \multicolumn{6}{|l|}{$\begin{array}{l}\text { Did you lose property because of the } \\
\text { floods in Fort McMurray? }\end{array}$} \\
\hline Home suffered substantial damage & $0(0.0)$ & $2(3.5)$ & $2(3.6)$ & $5(11.6)$ & $9(4.8)$ \\
\hline Home suffered slight damage & $0(0.0)$ & $2(3.5)$ & $2(3.6)$ & $1(2.3)$ & $5(2.7)$ \\
\hline Car was destroyed by the floods & $0(0.0)$ & $0(0.0)$ & $1(1.8)$ & $3(7.0)$ & $4(2.2)$ \\
\hline Business was destroyed by the floods & $0(0.0)$ & $3(5.3)$ & $2(3.6)$ & $1(2.3)$ & $6(3.2)$ \\
\hline Suffered no loss of property in the floods & $31(100.0)$ & $50(87.7)$ & $49(89.1)$ & $35(81.4)$ & $165(88.7)$ \\
\hline \multicolumn{6}{|l|}{$\begin{array}{l}\text { Do you live in the same house you lived } \\
\text { in before the floods? }\end{array}$} \\
\hline Same house & $21(80.8)$ & $47(88.7)$ & $40(78.4)$ & $39(90.7)$ & $147(85.0)$ \\
\hline $\begin{array}{l}\text { Different house-previous home not } \\
\text { destroyed by the fire }\end{array}$ & $5(19.2)$ & $5(9.4)$ & $8(15.7)$ & $4(9.3)$ & $22(12.7)$ \\
\hline $\begin{array}{l}\text { Different home-previous home } \\
\text { destroyed by the flood }\end{array}$ & $0(0.0)$ & $1(1.9)$ & $3(5.9)$ & $0(0.0)$ & $4(2.3)$ \\
\hline \multicolumn{6}{|l|}{ Support received from family } \\
\hline Some-to-high level of support & $17(65.4)$ & $34(65.4)$ & $25(52.1)$ & $24(58.5)$ & $100(59.9)$ \\
\hline Limited or no support & $9(34.6)$ & $18(34.6)$ & $23(47.9)$ & $17(41.5)$ & $67(40.1)$ \\
\hline \multicolumn{6}{|l|}{ Support received from Red Cross } \\
\hline Some-to-high level of support & $3(11.5)$ & $3(5.7)$ & $6(12.2)$ & $7(16.3)$ & $19(11.1)$ \\
\hline Limited or no support & $1(3.8)$ & $3(5.7)$ & $7(14.3)$ & $6(14.0)$ & $17(9.9)$ \\
\hline NA (not impacted by the flood) & $22(84.6)$ & $47(88.7)$ & $36(73.5)$ & $30(69.8)$ & $135(78.9)$ \\
\hline \multicolumn{6}{|l|}{$\begin{array}{l}\text { Support received from the Government } \\
\text { of Alberta }\end{array}$} \\
\hline Some-to-high level of support & $4(15.4)$ & $2(3.8)$ & $7(14.3)$ & $5(11.6)$ & $18(10.5)$ \\
\hline Limited or no support & $0(0.0)$ & $4(7.5)$ & $6(12.2)$ & $8(18.6)$ & $18(10.5)$ \\
\hline NA (not impacted by the flood) & $24(92.3)$ & 47 (88.7) & $36(73.5)$ & $30(69.8)$ & $135(78.9)$ \\
\hline \multicolumn{6}{|l|}{ Support received from Insurer } \\
\hline Some-to-high level of support & $2(7.7)$ & $2(3.8)$ & $2(4.1)$ & $4(9.3)$ & $10(5.8)$ \\
\hline Limited or no support & $0(0.0)$ & $4(7.5)$ & $7(14.3)$ & $7(16.3)$ & $18(10.5)$ \\
\hline NA (not impacted by the flood) & $24(92.3)$ & $47(88.7)$ & $40(81.6)$ & $32(74.4)$ & 143 (83.6 \\
\hline
\end{tabular}

MH: Mental health.

Regarding the flooding-related and received support information as mentioned above, 94.6\% (176) of the respondents resided at FMM during the 2020 flood; $17.6 \%$ (31) were living in the flooded areas; respondents reported receiving a some-to-high level of support 
from family were 59.9\% (100); from the Red Cross were 11.1\% (19); from the Government of Alberta were $10.5 \%$ (18); from the insurer were 5.8\% (10). More detailed characteristics of respondents are presented in Table 1.

\subsection{Univariate Analysis}

Associations between demographic, clinical antecedents, flooding-related experiences, and the likely PTSD were illustrated with the results of the univariate analysis in Table 2. We entered 41 predictor variables into the Chi-squared or Fisher exact test and found 14 predictor variables were significantly related to likely PTSD. Respondents who were not employed $(100.0 \%(8))$ were more likely to present with a likely PTSD compared to those who were employed $(36.5 \%(57))$. For the clinical characteristics, respondents with a history of depression $(67.3 \%(35))$, an anxiety disorder $(59.4 \%(41))$, or who had a history of mental health diagnosis before the flood $(54.8 \%$ (46)) were more likely to present with likely PTSD compared to respondents with no history of depression $(26.8 \%$ (30)), an anxiety disorder $(25.3 \%(24))$ or no mental health diagnosis history $(23.8 \%(19))$, respectively. Similarly, respondents who had a history of mental health counseling in the past year $(62.3 \%(38))$ and who would like to have mental health counseling $(59.3 \%$ (51)) were more likely to show PTSD symptoms than those who did not have a history of mental health counseling (26.2\% (27)) and those who would not like to receive mental health counseling (17.9\% (14)). Furthermore, respondents who were on antidepressants (56.9\% (29)), benzodiazepines $(100.0 \%(4))$, sleeping tablets $(76.5 \%(13))$, or who were on any medication for mental health concerns before the flood (53.4\% (31)) were more likely to develop PTSD compared to respondents who were not on antidepressants (31.9\% (36)), benzodiazepines (38.1\% (61)), sleeping tablets $(35.4 \%(52))$ or no psychotropic medication $(46.6 \%(27))$, respectively.

Table 2. Chi-squared test of association between demographic, clinical antecedents \& likely PTSD.

\begin{tabular}{|c|c|c|c|c|c|}
\hline Variables & $\begin{array}{l}\text { Unlikely } \\
\text { PTSD }\end{array}$ & Likely PTSD & $\begin{array}{c}\text { Chi-Square/Fisher } \\
\text { Exact }\end{array}$ & $p$-Value & Phi Value \\
\hline \multicolumn{6}{|c|}{ Demographic Characteristics } \\
\hline \multicolumn{6}{|l|}{ Gender } \\
\hline Male & $15(68.2)$ & $7(31.8)$ & & & \\
\hline Female & $84(59.2)$ & $58(40.8)$ & 0.65 & 0.49 & 0.06 \\
\hline \multicolumn{6}{|l|}{ Age categories } \\
\hline$\leq 40 y$ & $44(58.7)$ & $31(41.3)$ & & & \\
\hline$>40 \mathrm{y}$ & $55(61.8)$ & $34(38.2)$ & 0.17 & 0.75 & -0.03 \\
\hline \multicolumn{6}{|l|}{ Employment status } \\
\hline Employed & $99(63.5)$ & $57(36.5)$ & & & \\
\hline Unemployed & $0(0.0)$ & $8(100)$ & 12.81 & $<0.001^{*}$ & 0.28 \\
\hline \multicolumn{6}{|l|}{ Employment place } \\
\hline School boards & $54(72.0)$ & $21(28.0)$ & & & \\
\hline Healthcare industry & $5(62.5)$ & $3(37.5)$ & & & \\
\hline Keyano College & $12(60.0)$ & $8(40.0)$ & & & \\
\hline Oil Sands industry & $5(41.7)$ & $7(58.3)$ & & & \\
\hline Municipal or government agency & $8(66.7)$ & $4(33.3)$ & & & \\
\hline Other & $14(50.0)$ & $14(50.0)$ & 7.14 & 0.21 & 0.22 \\
\hline \multicolumn{6}{|l|}{ Relationship } \\
\hline In a relationship & $76(63.3)$ & $44(36.7)$ & & & \\
\hline Not in a relationship & $23(52.3)$ & $21(47.7)$ & 1.65 & 0.21 & 0.10 \\
\hline
\end{tabular}


Table 2. Cont.

\begin{tabular}{|c|c|c|c|c|c|}
\hline Variables & $\begin{array}{l}\text { Unlikely } \\
\text { PTSD }\end{array}$ & Likely PTSD & $\begin{array}{l}\text { Chi-Square/Fisher } \\
\text { Exact }\end{array}$ & $p$-Value & Phi Value \\
\hline \multicolumn{6}{|c|}{ Clinical Characteristics } \\
\hline \multicolumn{6}{|c|}{$\begin{array}{l}\text { History of Depression diagnosis from a } \\
\text { health professional? }\end{array}$} \\
\hline No & $82(73.2)$ & $30(26.8)$ & & & \\
\hline Yes & $17(32.7)$ & $35(67.3)$ & 24.37 & $<0.001 *$ & 0.39 \\
\hline \multicolumn{6}{|c|}{$\begin{array}{l}\text { History of Bipolar diagnoses from a } \\
\text { health professional? }\end{array}$} \\
\hline No & $96(60.4)$ & $63(39.6)$ & & & \\
\hline Yes & $3(60.0)$ & $2(40.0)$ & 0.00 & 0.99 & -0.00 \\
\hline \multicolumn{6}{|c|}{$\begin{array}{l}\text { History of Anxiety diagnosis from a } \\
\text { health professional? }\end{array}$} \\
\hline No & $71(74.7)$ & $24(25.3)$ & & & \\
\hline Yes & $28(40.6)$ & $41(59.4)$ & 19.49 & $<0.001 *$ & 0.35 \\
\hline \multicolumn{6}{|c|}{$\begin{array}{l}\text { History of Alcohol Abuse diagnosis } \\
\text { from a health professional? }\end{array}$} \\
\hline No & $97(60.2)$ & $64(39.8)$ & & & \\
\hline Yes & $2(66.7)$ & $1(33.3)$ & 0.05 & 0.99 & 0.02 \\
\hline \multicolumn{6}{|c|}{$\begin{array}{l}\text { History of Drug Abuse diagnosis from a } \\
\text { health professional? }\end{array}$} \\
\hline No & $98(60.5)$ & $64(39.5)$ & & & \\
\hline Yes & $1(50.0)$ & $1(50.0)$ & 0.09 & 0.99 & -0.02 \\
\hline \multicolumn{6}{|c|}{$\begin{array}{c}\text { History of Personality Disorder } \\
\text { diagnosis from a health professional? }\end{array}$} \\
\hline No & $98(60.1)$ & $65(39.9)$ & & & \\
\hline Yes & $1(100.0)$ & $0(0.0)$ & 0.66 & 0.99 & 0.06 \\
\hline \multicolumn{6}{|c|}{$\begin{array}{l}\text { History of Other diagnosis from a } \\
\text { health professional? }\end{array}$} \\
\hline No & $90(60.4)$ & $59(39.6)$ & & & \\
\hline Yes & $9(60.0)$ & $6(40.0)$ & 0.00 & 0.99 & -0.00 \\
\hline \multicolumn{6}{|c|}{$\begin{array}{c}\text { Never received a mental health } \\
\text { diagnosis from a health professional }\end{array}$} \\
\hline No & $61(76.3)$ & $19(23.8)$ & & & \\
\hline Yes, received MH Dx & $38(45.2)$ & $46(54.8)$ & 16.47 & $<0.001$ * & -0.32 \\
\hline \multicolumn{6}{|c|}{$\begin{array}{l}\text { History of mental health counseling in } \\
\text { the past year }\end{array}$} \\
\hline No & $76(73.8)$ & $27(26.2)$ & & & \\
\hline Yes & $23(37.7)$ & $38(62.3)$ & 20.84 & $<0.001$ * & 0.36 \\
\hline \multicolumn{6}{|c|}{$\begin{array}{l}\text { Would you like to receive mental health } \\
\text { counseling? }\end{array}$} \\
\hline No & $64(82.1)$ & $14(17.9)$ & & & \\
\hline Yes & $35(40.7)$ & $51(59.3)$ & 29.23 & $<0.001 *$ & 0.42 \\
\hline \multicolumn{6}{|c|}{ History of Antidepressant medications } \\
\hline No & $77(68.1)$ & $36(31.9)$ & & & \\
\hline Yes & $22(43.1)$ & $29(56.9)$ & 9.18 & $<0.01$ * & -0.24 \\
\hline \multicolumn{6}{|c|}{ History of Antipsychotic medications } \\
\hline No & $98(60.5)$ & $64(39.5)$ & & & \\
\hline Yes & $1(50.0)$ & $1(50.0)$ & 0.09 & 0.99 & 0.02 \\
\hline
\end{tabular}


Table 2. Cont.

\begin{tabular}{|c|c|c|c|c|c|}
\hline Variables & $\begin{array}{l}\text { Unlikely } \\
\text { PTSD }\end{array}$ & Likely PTSD & $\begin{array}{l}\text { Chi-Square/Fisher } \\
\text { Exact }\end{array}$ & $p$-Value & Phi Value \\
\hline \multicolumn{6}{|c|}{ Clinical Characteristics } \\
\hline \multicolumn{6}{|c|}{ History of Benzodiazepine medications } \\
\hline No & $99(61.9)$ & $61(38.1)$ & & & \\
\hline Yes & $0(0.0)$ & $4(100.0)$ & 6.25 & $0.02 *$ & 0.20 \\
\hline \multicolumn{6}{|c|}{ History of Mood Stabilizer medications } \\
\hline No & $95(61.3)$ & $60(38.7)$ & & & \\
\hline Yes & $4(44.4)$ & $5(55.6)$ & 1.01 & 0.49 & 0.08 \\
\hline \multicolumn{6}{|l|}{ History of Sleeping Tablets } \\
\hline No & $95(64.6)$ & $52(35.4)$ & & & \\
\hline Yes & $4(23.5)$ & $13(76.5)$ & 10.76 & $\leq 0.001$ * & 0.26 \\
\hline \multicolumn{6}{|c|}{$\begin{array}{l}\text { History of Other mental health } \\
\text { medications not listed }\end{array}$} \\
\hline No & $96(59.6)$ & $65(40.4)$ & & & \\
\hline Yes & $3(100.0)$ & $0(0.0)$ & 2.00 & 0.28 & -0.11 \\
\hline \multicolumn{6}{|c|}{$\begin{array}{l}\text { Not on any medication for mental } \\
\text { health concerns }\end{array}$} \\
\hline No & $72(67.9)$ & $34(32.1)$ & & & \\
\hline Yes, on MH Mx & $27(46.6)$ & $31(53.4)$ & 7.16 & $0.01 *$ & -0.21 \\
\hline \multicolumn{6}{|c|}{ Fort McMurray Flood Related Characteristics } \\
\hline \multicolumn{6}{|c|}{$\begin{array}{l}\text { Did you reside at Fort McMurray during } \\
\text { the } 2020 \text { flood? }\end{array}$} \\
\hline No & $4(66.7)$ & $2(33.3)$ & & & \\
\hline Yes & $95(60.1)$ & $63(39.9)$ & 0.10 & 0.99 & 0.03 \\
\hline \multicolumn{6}{|c|}{ Areas of residence during the 2020 flood } \\
\hline Not in the flooding area & $83(64.3)$ & $46(35.7)$ & & & \\
\hline In the flooding area & $12(41.4)$ & $17(58.6)$ & 5.20 & $0.04^{*}$ & 0.18 \\
\hline \multicolumn{6}{|l|}{ Housing status prior flood } \\
\hline Own home & $80(63.5)$ & $46(36.5)$ & & & \\
\hline Renting & $19(50.0)$ & $19(50.0)$ & 2.22 & 0.19 & 0.12 \\
\hline \multicolumn{6}{|l|}{ Housing status now } \\
\hline Own home & $78(60.0)$ & $52(40.0)$ & & & \\
\hline Renting & $21(61.8)$ & $13(38.2)$ & 0.04 & 0.99 & -0.02 \\
\hline \multicolumn{6}{|c|}{$\begin{array}{l}\text { Where did you live just prior to the } 2020 \\
\text { flooding? }\end{array}$} \\
\hline In Fort McMurray & $95(60.1)$ & $63(39.9)$ & & & \\
\hline Other & $4(66.7)$ & $2(33.3)$ & 0.10 & 0.99 & -0.03 \\
\hline \multicolumn{6}{|c|}{$\begin{array}{l}\text { Did you witness the flooding of any } \\
\text { homes or structures in Fort McMurray? }\end{array}$} \\
\hline No & $25(62.5)$ & $15(37.5)$ & & & \\
\hline Yes & $74(59.7)$ & $50(40.3)$ & 0.10 & 0.85 & 0.03 \\
\hline \multicolumn{6}{|c|}{$\begin{array}{l}\text { During the flooding, were you fearful } \\
\text { for your life or the lives of your friends } \\
\text { or family? }\end{array}$} \\
\hline No & $74(64.3)$ & $41(35.7)$ & & & \\
\hline Yes & $25(51.0)$ & $24(49.0)$ & 2.55 & 0.12 & 0.13 \\
\hline
\end{tabular}


Table 2. Cont.

\begin{tabular}{|c|c|c|c|c|c|}
\hline Variables & $\begin{array}{l}\text { Unlikely } \\
\text { PTSD }\end{array}$ & Likely PTSD & $\begin{array}{l}\text { Chi-Square/Fisher } \\
\text { Exact }\end{array}$ & $p$-Value & Phi Value \\
\hline \multicolumn{6}{|c|}{ Fort McMurray Flood Related Characteristics } \\
\hline \multicolumn{6}{|l|}{$\begin{array}{l}\text { During the } 2020 \text { Fort McMurray } \\
\text { flooding, how frequently did you watch } \\
\text { television images about the devastation } \\
\text { caused by the flood? }\end{array}$} \\
\hline Daily & $61(56.0)$ & $48(44.0)$ & & & \\
\hline$<$ Daily & $25(71.4)$ & $10(28.6)$ & & & \\
\hline $\begin{array}{c}\text { I did not watch the TV images of the } \\
\text { devastation. }\end{array}$ & $13(65.0)$ & $7(35.0)$ & 2.85 & 0.24 & 0.13 \\
\hline \multicolumn{6}{|l|}{$\begin{array}{l}\text { During the } 2020 \text { Fort McMurray } \\
\text { flooding, how frequently did you read } \\
\text { newspaper and internet articles related } \\
\text { to the devastation caused by the } \\
\text { flooding? }\end{array}$} \\
\hline Daily & $73(57.9)$ & $53(42.1)$ & & & \\
\hline$<$ Daily & $22(66.7)$ & $11(33.3)$ & & & \\
\hline $\begin{array}{l}\text { I did not read newspaper and internet } \\
\text { articles related to the devastation. }\end{array}$ & $4(80.0)$ & $1(20.0)$ & 1.66 & 0.40 & 0.10 \\
\hline \multicolumn{6}{|l|}{$\begin{array}{l}\text { Respondents who report their home } \\
\text { suffered substantial damage because of } \\
\text { the floods in Fort McMurray }\end{array}$} \\
\hline Unchecked & $98(63.2)$ & $57(36.8)$ & & & \\
\hline Checked & $1(11.1)$ & $8(88.9)$ & 9.66 & $<0.01 *$ & 0.24 \\
\hline \multicolumn{6}{|l|}{$\begin{array}{l}\text { Respondents who report their home } \\
\text { suffered slightly damage because of the } \\
\text { floods in Fort McMurray }\end{array}$} \\
\hline Unchecked & $98(61.6)$ & $61(38.4)$ & & & \\
\hline Checked & $1(20.0)$ & $4(80.0)$ & 3.51 & 0.08 & 0.15 \\
\hline \multicolumn{6}{|l|}{$\begin{array}{l}\text { Respondents report their car was } \\
\text { completely destroyed by the floods in } \\
\text { Fort McMurray }\end{array}$} \\
\hline Unchecked & $96(60.0)$ & $64(40.0)$ & & & \\
\hline Checked & $3(75.0)$ & $1(25.0)$ & 0.37 & 0.65 & -0.05 \\
\hline \multicolumn{6}{|l|}{$\begin{array}{l}\text { Respondents report their business was } \\
\text { completely destroyed by the floods in } \\
\text { Fort McMurray }\end{array}$} \\
\hline Unchecked & $95(59.7)$ & $64(40.3)$ & & & \\
\hline Checked & $4(80.0)$ & $1(20.0)$ & 0.83 & 0.65 & -0.07 \\
\hline \multicolumn{6}{|l|}{$\begin{array}{l}\text { Respondents report they suffered no } \\
\text { loss of property in the floods }\end{array}$} \\
\hline No, did not lose & $92(63.9)$ & $52(36.1)$ & & & \\
\hline Yes, lose & $7(35.0)$ & $13(65.0)$ & 6.12 & $0.02 *$ & 0.19 \\
\hline \multicolumn{6}{|l|}{$\begin{array}{c}\text { Do you live in the same house you lived } \\
\text { in before the floods? }\end{array}$} \\
\hline Yes & $86(61.9)$ & $53(38.1)$ & & & \\
\hline $\begin{array}{l}\text { No, I live in a different house even though } \\
\text { my previous home was not destroyed by } \\
\text { the flood. }\end{array}$ & $11(55.0)$ & $9(45.0)$ & & & \\
\hline $\begin{array}{l}\text { No, I live in a different house because my } \\
\text { previous home was destroyed by } \\
\text { the flood. }\end{array}$ & $1(25.0)$ & $3(75.0)$ & 2.45 & 0.35 & 0.12 \\
\hline
\end{tabular}


Table 2. Cont.

\begin{tabular}{|c|c|c|c|c|c|}
\hline Variables & $\begin{array}{l}\text { Unlikely } \\
\text { PTSD }\end{array}$ & Likely PTSD & $\begin{array}{c}\text { Chi-Square/Fisher } \\
\text { Exact }\end{array}$ & $p$-Value & Phi Value \\
\hline \multicolumn{6}{|c|}{ Fort McMurray Flood Related Characteristics } \\
\hline \multicolumn{6}{|l|}{ Support received from Family } \\
\hline Some-to-high level of support & $64(66.7)$ & $32(33.3)$ & & & \\
\hline Limited or not support & $30(48.4)$ & $32(51.6)$ & 5.22 & $0.03 *$ & 0.18 \\
\hline \multicolumn{6}{|l|}{ Support received from Red Cross } \\
\hline Some-to-high level of support & $8(47.1)$ & $9(52.9)$ & & & \\
\hline Limited or not support & $8(47.1)$ & $9(52.9)$ & 2.94 & 0.23 & 0.14 \\
\hline \multicolumn{6}{|l|}{$\begin{array}{c}\text { Support received from Government } \\
\text { of Alberta }\end{array}$} \\
\hline Some-to-high level of support & $7(41.2)$ & $10(58.8)$ & & & \\
\hline Limited or not support & $9(52.9)$ & $8(47.1)$ & 3.43 & 0.18 & 0.15 \\
\hline \multicolumn{6}{|l|}{ Support received from Insurer } \\
\hline \multicolumn{6}{|l|}{ Some-to-high level of support } \\
\hline Limited or not support & $5(50.0)$ & $5(50.0)$ & & & \\
\hline NA (not impacted by the flood) & $6(35.3)$ & $11(64.7)$ & 5.5 & 0.06 & 0.18 \\
\hline
\end{tabular}

$* p \leq 0.05$.

Considering the disaster-related information, respondents who lived in the flooded area during the 2020 floods (58.6\% (17)), who reported that their home suffered substantial damage because of the floods $(88.9 \%(8))$, and those who suffered loss of property in the floods $(65.0 \%$ (13)) were more likely to present with probable PTSD compared to those who did not live in the flooding area during the 2020 floods (35.7\% (46)), who did not suffer substantial damage because of the floods (36.8\% (57)), and who suffered no loss of property in the floods $(36.1 \%(52))$. Finally, respondents who reported that they received no support from family (51.6\% (32)) were more likely to present with PTSD compared to respondents who reported that they received some-to-high level of support from family (33.3\% (32)), respectively. In addition, we found two variables approaching significance. Respondents who reported that their home suffered slight damage because of the floods $(80.0 \%$ (4)) were more likely to report PTSD symptoms than those who reported that their home did not suffer any damage because of the flood (38.4\% (61)). Respondents who reported receiving no support from their insurer $(64.7 \%$ (11)) were more likely to present with probable PTSD compared to those who reported that they received a some-to-high level of support from the insurer $(50.0 \%(5))$.

\subsection{Logistic Regression}

Table 3 illustrates the multivariate logistic regression model that predicts likely PTSD among study participants. In total, 16 predictors were found with significant or nearsignificant $p$ values $(p \leq 0.1)$ (Table 2$)$. Never received a mental health diagnosis from a health professional, history of antidepressant medications, and suffered no loss of property in the floods were dropped from the regression model, as they were highly positively correlated with other variables ( $r s>0.7$ ). Therefore, we ended up entering 13 variables into the logistic regression model to predict the likelihood of PTSD.

The model was statistically significant; $\mathrm{X} 2(\mathrm{df}=14 ; \mathrm{n}=152)=84.98, p \leq 0.001$, suggesting that the model could distinguish between respondents who did or did not exhibit likely PTSD one year after the 2020 floods in FMM. The model explained 42.8\% (Cox and Snell R2) and 57.8\% (Nagelkerke R2) of the variance in indicating the likelihood that respondents will present with PTSD and correctly classified $80.3 \%$ of cases. 
Table 3. Logistic regression predicting likelihood of residents presenting with PTSD.

\begin{tabular}{|c|c|c|c|c|c|c|c|c|}
\hline \multirow{2}{*}{ Predictor } & \multirow{2}{*}{ B } & \multirow{2}{*}{ S.E. } & \multirow{2}{*}{ Wald } & \multirow{2}{*}{ df } & \multirow{2}{*}{$p$ Value } & \multirow{2}{*}{$\begin{array}{l}\text { Odds } \\
\text { Ratio }\end{array}$} & \multicolumn{2}{|c|}{ 95\% CI for Odds Ratio } \\
\hline & & & & & & & \multicolumn{2}{|c|}{ Upper Lower } \\
\hline Currently employed & -22.24 & $12,156.65$ & 0.00 & 1 & 0.99 & 0.00 & 0.00 & . \\
\hline $\begin{array}{c}\text { Having received Depression } \\
\text { diagnosis from a health professional }\end{array}$ & 1.74 & 0.62 & 7.82 & 1 & $0.01 *$ & 5.71 & 1.68 & 19.36 \\
\hline $\begin{array}{l}\text { Having received Anxiety diagnosis } \\
\text { from a health professional }\end{array}$ & 0.90 & 0.59 & 2.34 & 1 & 0.13 & 2.47 & 0.78 & 7.86 \\
\hline $\begin{array}{l}\text { Having received mental health } \\
\text { counseling in the past year }\end{array}$ & 0.92 & 0.58 & 2.54 & 1 & 0.11 & 2.51 & 0.81 & 7.78 \\
\hline $\begin{array}{l}\text { Would like to receive mental health } \\
\text { counseling }\end{array}$ & 1.06 & 0.53 & 4.02 & 1 & $0.05 *$ & 2.87 & 1.02 & 8.05 \\
\hline $\begin{array}{l}\text { On Benzodiazepines for a mental } \\
\text { health concern }\end{array}$ & -20.16 & $15,770.89$ & 0.00 & 1 & 0.99 & 0.00 & 0.00 & . \\
\hline On Sleeping Tablets & -0.99 & 0.97 & 1.05 & 1 & 0.31 & 0.37 & 0.06 & 2.48 \\
\hline $\begin{array}{c}\text { Not on any medication for a mental } \\
\text { health concern }\end{array}$ & 1.17 & 0.70 & 2.78 & 1 & 0.09 & 3.22 & 0.81 & 13.00 \\
\hline $\begin{array}{c}\text { Live in the area of flooding during } \\
\text { the } 2020 \text { flood }\end{array}$ & 0.11 & 0.80 & 0.02 & 1 & 0.89 & 1.11 & 0.23 & 5.36 \\
\hline $\begin{array}{c}\text { Home suffered substantial damage } \\
\text { as a result of the floods in Fort } \\
\text { McMurray }\end{array}$ & 3.42 & 2.19 & 2.44 & 1 & 0.12 & 30.69 & 0.42 & 2250.16 \\
\hline $\begin{array}{l}\text { Home suffered slight damage as a } \\
\text { result of the floods in Fort McMurray. }\end{array}$ & 2.18 & 1.60 & 1.84 & 1 & 0.18 & 8.82 & 0.38 & 204.61 \\
\hline $\begin{array}{l}\text { Received limited or no support from } \\
\text { family }\end{array}$ & 1.05 & 0.51 & 4.33 & 1 & $0.04 *$ & 2.87 & 1.06 & 7.74 \\
\hline $\begin{array}{l}\text { Received some-to-high level of } \\
\text { support from insurer }\end{array}$ & & & 1.65 & 2 & 0.44 & & & \\
\hline $\begin{array}{c}\text { Received limited or no support from } \\
\text { insurer }\end{array}$ & 2.03 & 1.70 & 1.43 & 1 & 0.23 & 7.61 & 0.27 & 213.07 \\
\hline Not impact by the flood & 1.35 & 1.67 & 0.65 & 1 & 0.42 & 3.84 & 0.15 & 101.93 \\
\hline Constant & 38.03 & $19,912.434$ & 0.000 & 1 & 0.99 & $3.30 \times 10^{16}$ & & \\
\hline
\end{tabular}

* Significance at $p \leq 0.05$ CI: confidence interval. S.E.: standard error. df: degree of freedom.

Among all the variables, only three of the predictors, having received depression diagnosis from a health professional, would like to receive mental health counseling and received limited or no support from family exhibited significant prediction of likely PTSD in the model. Respondents who reported that they received a depression diagnosis from a health professional were more than five times more likely to report likely PTSD symptoms because of floods, compared to those who did not $(\mathrm{OR}=5.71 ; 95 \% \mathrm{CI}$ : 1.68-19.36) while controlling for the other variables. Similarly, respondents who reported they would like to receive mental health counseling were almost three times more likely to report likely PTSD symptoms after floods, compared to those who were not willing to receive mental health counseling $(\mathrm{OR}=2.87 ; 95 \% \mathrm{CI}$ : 1.02-8.05), after controlling for other predictors. Moreover, respondents who reported that they received limited or no support from their family were almost three times more likely to express likely PTSD symptoms than those who reported receiving some-to-high levels of support from their families $(\mathrm{OR}=2.87$; 95\% CI: 1.06-7.74), after controlling for other model variables. The largest contribution to the model was provided by the received depression diagnosis (Wald $=7.82$ ).

\section{Discussion}

Our research evaluated the prevalence and the potential risk factors of likely PTSD, one year after the FMM flooding in 2020 among residents of FMM who participated in the study. In this study, the prevalence of likely PTSD among respondents was $39.6 \%$. Unlike our stated hypothesis, this outcome is consistent with findings from previous studies which estimate the prevalence of PTSD to be $30-40 \%$ among direct victims, $10-20 \%$ among rescue workers, and 5-10\% in the general population of FMM $[9,34,42,43]$. What aligned with our prediction is that the prevalence of likely PTSD in our study (one year after the flood) was much higher when compared with the prevalence of $12.8 \%$ and $13.6 \%$ in the FMM residents 6 months and 18 months after a 2016 wildfire [24,41]. This might be explained, 
as our study was conducted among a population that has experienced multiple traumatic events over the past 5 years (2016's wildfire, 2020's COVID and Flood). Thus, there is a potential additive effect that may explain why the rate of PTSD was higher than with the previous studies on wildfires. The time of sampling may be another significant factor, where the current study was carried out during the continuous COVID-19 pandemic when there was still so much uncertainty, while the wildfire surveys were conducted at 6 and 18 months after the wildfires when there was no pandemic. Future studies that evaluate multiple traumas on the population would be expected to clarify this.

None of the demographic characteristics showed an association with likely PTSD after flooding. Although compared to males and older age groups, females and the younger age group expressed a higher prevalence of PTSD symptoms, the difference was slight and not significant. This conflicts with previous studies, where females and younger age groups were indicated to be more likely to express mental health problems, including likely PTSD symptoms, compared to males and older age groups [44-48]. Since our study sample size was relatively small, it may not have elicited that difference as reported in other studies.

Among clinical predictors, prior depression history was a significant risk factor for the likelihood of PTSD. The majority of respondents who had a depression history (67.3\%) reported having likely PTSD symptoms after the flood. This finding is highly consistent with research on the main effects of prior mental health disorders on PTSD [3,45,49-52]. Much research has determined a relationship between depression and PTSD among those who have experienced trauma, including natural disasters, childhood maltreatment, serious accident, or terrorist act [53-55]. Worthington's research had identified past borderline personality disorder diagnosis and past depression as two of the most significant prediction for the onset of PTSD after the disaster [45]. In addition, a study with 1820 respondents who supported the mission in Afghanistan [51] showed that the prevalence of PTSD was highest among those with a pre-deployment history of depression [51]. This might suggest that past psychiatric diagnoses such as depression may successfully predict future mental health disorders [51]. Likewise, in China, a cross-sectional study aimed to investigate people's mental health conditions during the COVID-19 pandemic reported that more than one-third of the study participants who met diagnostic criteria for PTSD had a psychiatric diagnosis history $[49,56]$. A similar result can also be found in a long-term study of the SARS-CoV-1 pandemic [50].

The connection between PTSD and the prior depression history could be interpreted in a number of ways [57]. One possibility is that people who have depression show a higher possibility to encounter traumatic experiences than those without depression, which, in turn, may improve the possibilities of the onset of PTSD [57]. The other explanation is about the gene. Family history is a significant predictor for the development of depression. Therefore, it makes sense that some specific genetic factors might play a role in the appearance of both depression and PTSD [5].

Family support was found to have a sign of significantly associated valence with likely PTSD. In our study, respondents who reported receiving limited or no support from their family were more likely to show PTSD symptoms than their counterparts who received some-to-high level of family support. Consistent with our finding, family supports have long been regarded as protective solid or resource factors in the aftermath of trauma. Sufficient social support, primarily coming from family, before the disaster, can reduce the adverse psychological effects of natural disaster exposure and improve coping and resilience [31,58-61]. Conversely, lack of social support in close family members friends before a traumatic event has been associated with the development of PTSD in numerous populations following the disaster [62-64]. Previous studies conducted in FMM after the devastating wildfire in 2016 indicated that having only limited or no support from friends and family is one of the significant risk factors for likely PTSD among the residents of FMM post-disaster $[9,43,65]$. This finding is consistent with the longitudinal study about crisis support following the MS Herald of Free Enterprise disaster $[9,66]$; the study aimed at exploring the coping strategies for technological disasters [67], the research about 
psychiatric disorders among victims of courthouse shootings [68], and the exploration about psychiatric disorders among survivors of the Oklahoma City Bombing [69].

The willingness to receive mental health counseling after the disaster was another predictor in our study. In published studies, there is not much data addressing the risk factors associated with post-disaster counselling. According to some research, counseling may not relieve symptoms of psychiatric disorders such as PTSD after disaster events [70]. For example, according to the 2002 Cochran review, post-trauma debriefing may offer no PTSD preventative benefits and might potentially be harmful [70,71]. Therefore, our finding may offer explanation as to why participants who experienced PTSD (low resilience) preferred to seek counseling assistance after experiencing intensive distress and mental health problems. In the future, studies with more concrete output on events pre and post disasters are required to indicate any negative relationship between seeking counseling and mental health outcomes.

\section{Limitations of the Study}

Our study has some limitations that need to be considered when interpreting the findings. Firstly, this study was conducted during the COVID-19 pandemic; thus, the panic might have contributed to the high PTSD symptoms in study participants. Moreover, the survey link was sent by the community providers; thus, there was no way of knowing the actual number of people who received the survey. Therefore, the response rate in our study may be overestimated, as it is based only on the number of people who clicked the survey link instead of the number of people who received the survey. Besides, our sample size of 186 was much less than the sample size estimates that we projected. As such, the margin error of our prevalence estimates was $\pm 7 \%$ rather than the $\pm 3 \%$, which we projected initially. Due to the unbalanced groups in our data, some variables were dropped from the analysis which might have confounded the results of this study. In addition, there was an over-representation of a history of mental health conditions in respondents in this study. In addition, all the symptom-related responses were self-reported and were not based on clinical assessment, which may undermine the validity of the study findings. Moreover, we could not collect data from all categories of FMM inhabitants, such as the workers in remote camps. Despite these limitations, our study may be considered as an accurate reflection of respondents from the sites sampled and, thus, useful for policy purposes.

\section{Conclusions}

This study has evaluated the prevalence of PTSD among the residents of FMM one year after the devastating flood and has determined the demographic, clinical, disaster-related, and other risk variables of likely PTSD in the respondents. Our findings indicated that one year after the FMM flood in 2020, 39.6\% of the respondents ( $40.8 \%$ females and $31.8 \%$ males) keep suffering from probable PTSD. Consistent with previous studies, perceived family and social support were one of the solid protective factors. It would be useful for future studies and policy practitioners to investigate more closely the interaction between emotional support that survivors can receive from family, friends and material support offered by public institutions or charities in the context of mental wellness and recovery. For example, the lessons learned from the $2016 \mathrm{FMM}$ wildfires were successfully applied to the 2018 wildfire in British Columbia. These were mainly about cooperation and communication among various relief organizations and more efforts to build community partnerships, which could improve mental health support during the recovery process for the affected communities [32]. In addition, receiving a mental health depression diagnosis from a health professional before the disaster and wanting to receive mental health counseling after the disaster were significant risk predictors for suffering from a likely PTSD. This information might have significant reference value for policymakers when crafting crisis response programs at a population level to alleviate the effects of natural disasters on the public's mental health. More in-depth studies in the future are required to explore the possible association between counseling and probable PTSD symptoms. On top of 
that, preparedness plans for future disasters might need to provide broad mental health resilience education to the residents in a high-risk area. A variety of innovative programs or mobile health options $[24,32,65]$ that can provide cost-effective, self-directed, and locationindependent interventions that can enhance accessibility and continuity of care in situations where resources are scare should be introduced to high-risk groups, in particular, to help policymakers effectively process the post-disaster mental help in the future.

Author Contributions: The study was conceived and designed by V.I.O.A. W.M. drafted the initial manuscript. R.S. conducted data analysis, contributed to writing, and critically reviewing the manuscript. E.E. contributed to the design of the study, writing, and critically reviewing the manuscript. N.N. contributed to writing and critical review of the manuscript. B.A. contributed to writing and critical review of the manuscript. H.P. contributed to writing and critical review of the manuscript. G.O.-D. contributed to writing and critical review of the manuscript. M.A. contributed to writing and critical review of the manuscript. E.O. contributed to writing and critical review of the manuscript. F.O. contributed to writing and critical review of the manuscript. Y.Z. contributed to writing and critical review of the manuscript. All authors have read and agreed to the published version of the manuscript.

Funding: This study was supported by grants from the Mental Health Foundation and the Douglas Harden Trust Fund.

Institutional Review Board Statement: The study approval was granted by the University of Alberta Research and Ethics Committee (Pro00066054).

Informed Consent Statement: Consent was implied if participants completed and submitted the online survey questionnaire.

Data Availability Statement: Data is available upon reasonable request to the submitting author.

Acknowledgments: Support with survey link distribution was received from the Fort McMurray Public and Catholic School Boards, Keyano College, and the Canadian Mental Health Association.

Conflicts of Interest: All the authors disclose no conflict of interest.

\section{References}

1. Leaning, J.; Guha-Sapir, D. Natural disasters, armed conflict, and public health. N. Engl. J. Med. 2013, 369, 1836-1842. [CrossRef] [PubMed]

2. Guha-Sapir, D.; Vos, F.; Below, R.; Ponserre, S. Annual Disaster Statistical Review 2011: The Numbers and Trends; Université Catholique de Louvain: Ottignies-Louvain-la-Neuve, Belgium, 2012.

3. Goldmann, E.; Galea, S. Mental health consequences of disasters. Annu. Rev. Public Health 2014, 35, 169-183. [CrossRef]

4. Noji, E.K. Natural disasters. Crit. Care Clin. 1991, 7, 271-292. [CrossRef]

5. WHO. Floods. 2021. Available online: https://www.who.int/health-topics/floods\#tab=tab_1 (accessed on 24 October 2021).

6. EM-DAT: The International Disaster Database. 2005. Available online: http:/ / www.em-dat.net/ (accessed on 24 October 2021).

7. Stanke, C.; Murray, V.; Amlôt, R.; Nurse, J.; Williams, R. The effects of flooding on mental health: Outcomes and recommendations from a review of the literature. PLoS Curr. 2012, 4, e4f9f1fa9c3cae. [CrossRef] [PubMed]

8. Agyapong, V.I.; Juhás, M.; Mrklas, K.; Hrabok, M.; Omeje, J.; Gladue, I.; Kozak, J.; Leslie, M. Randomized controlled pilot trial of supportive text messaging for alcohol use disorder patients. J. Subst. Abus. Treat. 2018, 94, 74-80. [CrossRef]

9. Brown, M.R.; Agyapong, V.; Greenshaw, A.J.; Cribben, I.; Brett-MacLean, P.; Drolet, J.; McDonald-Harker, C.; Omeje, J.; Mankowsi, M.; Noble, S.; et al. Significant PTSD and other mental health effects present 18 months after the Fort Mcmurray wildfire: Findings from 3070 grades 7-12 students. Front. Psychiatry 2019, 10, 623. [CrossRef] [PubMed]

10. Briere, J.; Elliott, D. Prevalence, characteristics, and long-term sequelae of natural disaster exposure in the general population. J. Trauma. Stress 2000, 13, 661-679. [CrossRef] [PubMed]

11. Laugharne, J.; Van de Watt, G.; Janca, A. After the fire: The mental health consequences of fire disasters. Curr. Opin. Psychiatry 2011, 24, 72-77. [CrossRef] [PubMed]

12. McFarlane, A.C.; Van Hooff, M. Impact of childhood exposure to a natural disaster on adult mental health: 20-year longitudinal follow-up study. Br. J. Psychiatry 2009, 195, 142-148. [CrossRef] [PubMed]

13. Morgan, L.; Scourfield, J.; Williams, D.; Jasper, A.; Lewis, G. The Aberfan disaster: 33-year follow-up of survivors. Br. J. Psychiatry 2003, 182, 532-536. [CrossRef]

14. Galea, S.; Nandi, A.; Vlahov, D. The epidemiology of post-traumatic stress disorder after disasters. Epidemiol. Rev. 2005, $27,78-91$. [CrossRef] [PubMed] 
15. Chen, L.; Liu, A. The incidence of posttraumatic stress disorder after floods: A meta-analysis. Disaster Med. Public Health Prep. 2015, 9, 329-333. [CrossRef]

16. Tapsell, S.M.; Tunstall, S.M. "I wish I'd never heard of Banbury": The relationship between 'place' and the health impacts from flooding. Health Place 2008, 14, 133-154. [CrossRef] [PubMed]

17. Carroll, B.; Balogh, R.; Morbey, H.; Araoz, G. Health and social impacts of a flood disaster: Responding to needs and implications for practice. Disasters 2010, 34, 1045-1063. [CrossRef] [PubMed]

18. Collins, T.W.; Jimenez, A.M.; Grineski, S.E. Hispanic health disparities after a flood disaster: Results of a population-based survey of individuals experiencing home site damage in El Paso (Texas, USA). J. Immigr. Minority Health 2013, 15, 415-426. [CrossRef]

19. Rosen, C.S.; Matthieu, M.M.; Norris, F.H. Factors predicting crisis counselor referrals to other crisis counseling, disaster relief, and psychological services: A cross-site analysis of post-Katrina programs. Adm. Policy Ment. Health Ment. Health Serv. Res. 2009, 36, 186-194. [CrossRef] [PubMed]

20. Lamond, J.E.; Joseph, R.D.; Proverbs, D.G. An exploration of factors affecting the long term psychological impact and deterioration of mental health in flooded households. Environ. Res. 2015, 140, 325-334. [CrossRef] [PubMed]

21. Shultz, J.M.; McLean, A.; Herberman Mash, H.B.; Rosen, A.; Kelly, F.; Solo-Gabriele, H.M.; Youngs, G.A., Jr.; Jensen, J.; Bernal, O.; Neria, Y. Mitigating flood exposure: Reducing disaster risk and trauma signature. Disaster Health 2013, 1, 30-44. [CrossRef]

22. Paranjothy, S.; Gallacher, J.; Amlôt, R.; Rubin, G.J.; Page, L.; Baxter, T.; Wight, J.; Kirrage, D.; McNaught, R.; Palmer, S.R. Psychosocial impact of the summer 2007 floods in England. BMC Public Health 2011, 11, 145. [CrossRef] [PubMed]

23. Census. Municipal Census Report. 2018. Available online: https://www.rmwb.ca/en/permits-and-development/resources/ Documents/Latest-Census-Report-2018.pdf (accessed on 18 October 2021).

24. Agyapong, V.I.; Juhas, M.; Omege, J.; Denga, E.; Nwaka, B.; Akinjise, I.; Corbett, S.E.; Brown, M.; Chue, P.; Li, X.M.; et al Prevalence rates and correlates of likely post-traumatic stress disorder in residents of fort mcmurray 6 months after a wildfire. Int. J. Ment. Health Addict. 2021, 19, 632-650. [CrossRef]

25. Bench, A. Fort McMurray Flood Caused \$228M in Insured Damages. 2020. Available online: https://globalnews.ca/news/7013 040/fort-mcmurray-flood-damages-insurance/ (accessed on 16 November 2021).

26. Bressler, R.; Erford, B.T.; Dean, S. A systematic review of the posttraumatic stress disorder checklist (PCL). J. Couns. Dev. 2018, 96, 167-186. [CrossRef]

27. Sanchez-Gomez, M.; Giorgi, G.; Finstad, G.L.; Urbini, F.; Foti, G.; Mucci, N.; Zaffina, S.; León-Perez, J.M. COVID-19 pandemic as a traumatic event and its associations with fear and mental health: A cognitive-activation approach. Int. J. Environ. Res. Public Health 2021, 18, 7422. [CrossRef] [PubMed]

28. Shalaby, R.; Eboreime, E.; Nkire, N.; Agyapong, B.; Pazderka, H.; Obuobi-Donkor, G.; Adu, M.K.; Mao, W.; Owusu, E.; Oluwasina F.; et al. COVID-19 Pandemic in a Vulnerable Population: Prevalence and Correlates of Anxiety. Behav. Sci. 2022, 12, 13. [CrossRef]

29. Malbeuf, J. Berms and Sandbags: Fort McMurray Spending Millions in Preparation for Flood Season. Available online: https: //www.cbc.ca/news/canada/edmonton/fort-mcmurray-flood-river-wood-buffalo-1.5956024 (accessed on 20 March 2021).

30. Brown, M.R.; Agyapong, V.; Greenshaw, A.J.; Cribben, I.; Brett-MacLean, P.; Drolet, J.; McDonald-Harker, C.; Omeje, J.; Mankowsi, M.; Noble, S.; et al. After the Fort McMurray wildfire there are significant increases in mental health symptoms in grade 7-12 students compared to controls. BMC Psychiatry 2019, 19, 18.

31. Mao, W.; Agyapong, V.I. The Role of Social Determinants in Mental Health and Resilience After Disasters: Implications for Public Health Policy and Practice. Front. Public Health 2021, 9, 658528. [CrossRef] [PubMed]

32. Agyapong, V.I.; Hrabok, M.; Juhas, M.; Omeje, J.; Denga, E.; Nwaka, B.; Akinjise, I.; Corbett, S.E.; Moosavi, S.; Brown, M.; et al Prevalence rates and predictors of generalized anxiety disorder symptoms in residents of Fort McMurray six months after a wildfire. Front. Psychiatry 2018, 9, 345. [CrossRef]

33. Harris, P.A.; Taylor, R.; Thielke, R.; Payne, J.; Gonzalez, N.; Conde, J.G. Research electronic data capture (REDCap)—A metadatadriven methodology and workflow process for providing translational research informatics support. J. Biomed. Inform. 2009, 42, 377-381. [CrossRef] [PubMed]

34. Swenson, C.C.; Saylor, C.F.; Powell, M.P.; Stokes, S.J.; Foster, K.Y.; Belter, R.W. Impact of a natural disaster on preschool children: Adjustment 14 months after a hurricane. Am. J. Orthopsychiatry 1996, 66, 122-130. [CrossRef]

35. Conybeare, D.; Behar, E.; Solomon, A.; Newman, M.G.; Borkovec, T. The PTSD Checklist—Civilian Version: Reliability, validity, and factor structure in a nonclinical sample. J. Clin. Psychol. 2012, 68, 699-713. [CrossRef]

36. Weathers, F.W.; Litz, B.T.; Herman, D.S.; Huska, J.A.; Keane, T.M. The PTSD Checklist (PCL): Reliability, validity, and diagnostic utility. Annu. Conv. Int. Soc. Trauma. Stress Stud. San Antonio 1993, 462.

37. Alhalal, E.; Ford-Gilboe, M.; Wong, C.; AlBuhairan, F. Reliability and validity of the Arabic PTSD Checklist Civilian Version (PCL-C) in women survivors of intimate partner violence. Res. Nurs. Health 2017, 40, 575-585. [CrossRef]

38. Reichenheim, M.E.; Oliveira, A.G.; Moraes, C.L.; Coutinho, E.S.; Figueira, I.; Lobato, G. Reappraising the dimensional structure of the PTSD Checklist: Lessons from the DSM-IV-based PCL-C. Braz. J. Psychiatry 2017, 40, 154-162. [CrossRef]

39. Kip, K.E.; Elk, C.A.; Sullivan, K.L.; Kadel, R.; Lengacher, C.A.; Long, C.J.; Rosenzweig, L.; Shuman, A.; Hernandez, D.F.; Street, J.D.; et al. Brief treatment of symptoms of post-traumatic stress disorder (PTSD) by use of accelerated resolution therapy (ART). Behav. Sci. 2012, 2, 115-134. [CrossRef] 
40. Kipa, K.E.; Rosenzweiga, L.; Hernandeza, D.F.; Shumana, A.; Witta, A.; Kellera, E.; Diamond, D.B. Accelerated Resolution Therapy: A Brief, Emerging Evidence-Based Treatment for PTSD. In Comprehensive Guide to Post-Traumatic Stress Disorder; Springer: Cham, Switzerland, 2015.

41. Norris, F.H.; Friedman, M.J.; Watson, P.J. 60,000 disaster victims speak: Part II. Summary and implications of the disaster mental health research. Psychiatry Interpers. Biol. Process. 2002, 65, 240-260. [CrossRef]

42. Stewart, S.H.; Mitchell, T.L.; Wright, K.D.; Loba, P. The relations of PTSD symptoms to alcohol use and coping drinking in volunteers who responded to the Swissair Flight 111 airline disaster. J. Anxiety Disord. 2004, 18, 51-68. [CrossRef]

43. Moosavi, S.; Nwaka, B.; Akinjise, I.; Corbett, S.E.; Chue, P.; Greenshaw, A.J.; Silverstone, P.H.; Li, X.M.; Agyapong, V.I. Mental health effects in primary care patients 18 months after a major wildfire in Fort McMurray: Risk increased by social demographic issues, clinical antecedents, and degree of fire exposure. Front. Psychiatry 2019, 10, 683. [CrossRef]

44. Nwachukwu, I.; Nkire, N.; Shalaby, R.; Hrabok, M.; Vuong, W.; Gusnowski, A.; Surood, S.; Urichuk, L.; Greenshaw, A.J.; Agyapong, V.I. COVID-19 pandemic: Age-related differences in measures of stress, anxiety and depression in Canada. Int. J. Environ. Res. Public Health 2020, 17, 6366. [CrossRef]

45. Worthington, M.A.; Mandavia, A.; Richardson-Vejlgaard, R. Prospective prediction of PTSD diagnosis in a nationally representative sample using machine learning. BMC Psychiatry 2020, 20, 532. [CrossRef]

46. Contractor, A.A.; Greene, T.; Dolan, M.; Elhai, J.D. Relations between PTSD and depression symptom clusters in samples differentiated by PTSD diagnostic status. J. Anxiety Disord. 2018, 59, 17-26. [CrossRef]

47. Andrews, B.; Brewin, C.R.; Rose, S. Gender, social support, and PTSD in victims of violent crime. J. Trauma. Stress 2003, 16, 421-427. [CrossRef]

48. Bokszczanin, A. PTSD symptoms in children and adolescents 28 months after a flood: Age and gender differences. J. Trauma. Stress Off. Publ. Int. Soc. Trauma. Stress Stud. 2007, 20, 347-351. [CrossRef] [PubMed]

49. Shalaby, R.; Adu, M.K.; Andreychuk, T.; Eboreime, E.; Gusnowski, A.; Vuong, W.; Surood, S.; Greenshaw, A.J.; Agyapong, V.I. Prevalence, Demographic, and Clinical Correlates of Likely PTSD in Subscribers of Text4Hope during the COVID-19 Pandemic. Int. J. Environ. Res. Public Health 2021, 18, 6227. [CrossRef] [PubMed]

50. Steardo, L.; Verkhratsky, A. Psychiatric face of COVID-19. Transl. Psychiatry 2020, 10, 261. [CrossRef] [PubMed]

51. Beliveau, P.J.; Sampasa-Kanyinga, H.; Colman, I.; Zamorski, M.A. Are personnel with a past history of mental disorders disproportionately vulnerable to the effects of deployment-related trauma? A cross-sectional study of Canadian military personnel. BMC Psychiatry 2019, 19, 156.

52. Maguen, S.; Neria, Y.; Conoscenti, L.M.; Litz, B.T. Depression and Prolonged Grief in the Wake of Disasters; American Psychological Association: Washington, DC, USA, 2012.

53. Shalev, A.Y.; Freedman, S.; Peri, T.; Brandes, D.; Sahar, T.; Orr, S.P.; Pitman, R.K. Prospective study of posttraumatic stress disorder and depression following trauma. Am. J. Psychiatry 1998, 155, 630-637. [CrossRef]

54. Vranceanu, A.-M.; Hobfoll, S.E.; Johnson, R.J. Child multi-type maltreatment and associated depression and PTSD symptoms: The role of social support and stress. Child Abus. Negl. 2007, 31, 71-84. [CrossRef]

55. Kilpatrick, D.G.; Ruggiero, K.J.; Acierno, R.; Saunders, B.E.; Resnick, H.S.; Best, C.L. Violence and risk of PTSD, major depression, substance abuse/dependence, and comorbidity: Results from the National Survey of Adolescents. J. Consult. Clin. Psychol. 2003, 71, 692. [CrossRef]

56. Hao, F.; Tan, W.; Jiang, L.; Zhang, L.; Zhao, X.; Zou, Y.; Hu, Y.; Luo, X.; Jiang, X.; McIntyre, R.S.; et al. Do psychiatric patients experience more psychiatric symptoms during COVID-19 pandemic and lockdown? A case-control study with service and research implications for immunopsychiatry. Brain Behav. Immun. 2020, 87, 100-106. [CrossRef]

57. Tull, M.T.; Vidaña, A.G.; Betts, J.E. Emotion Regulation Difficulties in PTSD, in Emotion in Posttraumatic Stress Disorder; Elsevier: Amsterdam, The Netherlands, 2020; pp. 295-310.

58. Sasaki, Y.; Tsuji, T.; Koyama, S.; Tani, Y.; Saito, T.; Kondo, K.; Kawachi, I.; Aida, J. Neighborhood ties reduced depressive symptoms in older disaster survivors: Iwanuma study, a natural experiment. Int. J. Environ. Res. Public Health 2020, 17, 337. [CrossRef]

59. Sasaki, Y.; Aida, J.; Tsuji, T.; Koyama, S.; Tsuboya, T.; Saito, T.; Kondo, K.; Kawachi, I. Pre-disaster social support is protective for onset of post-disaster depression: Prospective study from the Great East Japan Earthquake \& Tsunami. Sci. Rep. 2019, 9, 19427.

60. Chan, C.S.; Lowe, S.R.; Weber, E.; Rhodes, J.E. The contribution of pre-and postdisaster social support to short-and long-term mental health after Hurricanes Katrina: A longitudinal study of low-income survivors. Soc. Sci. Med. 2015, 138, 38-43. [CrossRef] [PubMed]

61. Lowe, S.R.; Chan, C.S.; Rhodes, J.E. Pre-hurricane perceived social support protects against psychological distress: A longitudinal analysis of low-income mothers. J. Consult. Clin. Psychol. 2010, 78, 551. [CrossRef] [PubMed]

62. Agaibi, C.E.; Wilson, J.P. Trauma, PTSD, and resilience: A review of the literature. Trauma Violence Abus. 2005, 6, 195-216. [CrossRef] [PubMed]

63. Laffaye, C.; Cavella, S.; Drescher, K.; Rosen, C. Relationships among PTSD symptoms, social support, and support source in veterans with chronic PTSD. J. Trauma. Stress Off. Publ. Int. Soc. Trauma. Stress Stud. 2008, 21, 394-401. [CrossRef] [PubMed]

64. Brewin, C.R.; Andrews, B.; Valentine, J.D. Meta-analysis of risk factors for posttraumatic stress disorder in trauma-exposed adults. J. Consult. Clin. Psychol. 2000, 68, 748. [CrossRef] 
65. Agyapong, V.I.; Mrklas, K.; Juhás, M.; Omeje, J.; Ohinmaa, A.; Dursun, S.M.; Greenshaw, A.J. Cross-sectional survey evaluating Text4Mood: Mobile health program to reduce psychological treatment gap in mental healthcare in Alberta through daily supportive text messages. BMC Psychiatry 2016, 16, 378. [CrossRef] [PubMed]

66. Dalgleish, T.; Joseph, S.; Thrasher, S.; Tranah, T.; Yule, W. Crisis support following the Herald of free-enterprise disaster: A longitudinal perspective. J. Trauma. Stress 1996, 9, 833-845. [CrossRef] [PubMed]

67. Arata, C.M.; Picou, J.S.; Johnson, G.D.; McNally, T.S. Coping with technological disaster: An application of the conservation of resources model to the Exxon Valdez oil spill. J. Trauma. Stress Off. Publ. Int. Soc. Trauma. Stress Stud. 2000, 13, 23-39. [CrossRef]

68. Johnson, S.D.; North, C.S.; Smith, E.M. Psychiatric disorders among victims of a courthouse shooting spree: A three-year follow-up study. Community Ment. Health J. 2002, 38, 181-194. [CrossRef] [PubMed]

69. North, C.S.; Tivis, L.; McMillen, J.C.; Pfefferbaum, B.; Cox, J.; Spitznagel, E.L.; Bunch, K.; Schorr, J.; Smith, E.M. Coping, functioning, and adjustment of rescue workers after the Oklahoma City bombing. J. Trauma. Stress Off. Publ. Int. Soc. Trauma. Stress Stud. 2002, 15, 171-175. [CrossRef]

70. Litz, B.T.; Gray, M.J.; Bryant, R.A.; Adler, A.B. Early intervention for trauma: Current status and future directions. Clin. Psychol. Sci. Pract. 2002, 9, 112. [CrossRef]

71. Rose, S.C.; Bisson, J.; Churchill, R.; Wessely, S. Psychological debriefing for preventing post traumatic stress disorder (PTSD). Cochrane Database Syst. 2002, 2, CD000560. [CrossRef] [PubMed] 